\title{
QUADRATIC AND QUASI-QUADRATIC FUNCTIONALS
}

\author{
PETER ŠEMRL
}

(Communicated by Maurice Auslander)

\begin{abstract}
In this note we show how Jordan *-derivations arise as a "measure" of the representability of quasi-quadratic functionals by sesquilinear ones. Our main result can be considered as an extension of the Jordan-von Neumann characterization of pre-Hilbert space.
\end{abstract}

\section{INTRODUCTION}

Let $M$ be a module over a $*$-ring $R$. A mapping $S: M \times M \rightarrow R$ is called a sesquilinear functional if it is linear in the first argument and antilinear in the second argument:

(1) $S(a x+b y, z)=a S(x, z)+b S(y, z), \quad x, y, z \in M, a, b \in R$, (2) $S(x, a y+b z)=S(x, y) a^{*}+S(x, z) b^{*}, \quad x, y, z \in M, a, b \in R$.

In the special case when $R$ is a commutative ring with the trivial involution $a^{*}=a$, the relation (2) can be rewritten as $S(x, a y+b z)=a S(x, y)+$ $b S(x, z)$. In this case the mapping $S$ is called bilinear.

A quadratic functional $Q$ on $M$ is defined as the composition of some sesquilinear functional from $M \times M$ to $R$ with the diagonal injection of $M$ into $M \times M$; that is, $Q(x)=S(x, x)$, where $S$ is sesquilinear. There is something inappropriate about defining a quadratic functional which is a function of one variable in terms of a sesquilinear functional which involves two variables. This raises the question of what requirements can be imposed on a mapping from $M$ to $R$ to define the set of all quadratic functionals. The best-known identities satisfied by quadratic functionals are the parallelogram law

$$
Q(x+y)+Q(x-y)=2 Q(x)+2 Q(y), \quad x, y \in M,
$$

and the homogeneity equation

$$
Q(a x)=a Q(x) a^{*}, \quad x \in M, a \in R .
$$

A mapping $Q: M \rightarrow R$ satisfying these two identities is called a quasi-quadratic functional. In the special case that $R$ is a commutative ring with the trivial involution the relation (4) can be rewritten as $Q(a x)=a^{2} Q(x)$.

Received by the editors August 27, 1991 and, in revised form, April 14, 1992.

1991 Mathematics Subject Classification. Primary 15A63, 16A28, 39B50.

This work was supported by the Research Council of Slovenia. 
It seems natural to ask when quasi-quadratic functionals are in fact quadratic functionals. In other words, given a quasi-quadratic functional $Q$, does there exist a sesquilinear functional $S$ such that $Q(x)=S(x, x)$ ? In 1963, Halperin in his lectures on Hilbert spaces posed this problem for the special case that $M$ is a vector space over $\mathbb{F} \in\{\mathbb{R}, \mathbb{C}, \mathbb{H}\}$. Here, $\mathbb{R}$ and $\mathbb{C}$ denote the field of real numbers and the field of complex numbers respectively, while $\mathbb{H}$ denotes the skew-field of quaternions. In 1964, Kurepa [4] obtained the general form of quasi-quadratic functionals defined on a vector space over $\mathbb{R}$. In particular, he showed that there exist quasi-quadratic functionals which cannot be represented by bilinear functionals. In 1966, Gleason [2] generalized this result to vector spaces $V, \operatorname{dim} V \geq 2$, over an arbitrary field $F$, not of characteristic 2 , and with the trivial involution. He proved that all quasi-quadratic functionals on $V$ are quadratic if and only if all additive derivations on $F$ are zero. The same result holds for quasi-quadratic functionals defined on a module over a commutative ring $R$ with the trivial involution in which 2 is a unit. This result follows from [1, Theorem 3]. It should be mentioned that in this commutative case with the trivial involution the result of Jordan and von Neumann [3] implies that for each quasi-quadratic functional $Q$ the mapping $S$ defined by

$$
4 S(x, y)=Q(x+y)-Q(x-y)
$$

is symmetric and biadditive and $Q(x)=S(x, x)$ (see [2]). Thus, the abovementioned results imply that $S$ is homogeneous in both variables if and only if all additive derivations on $R$ are zero.

In 1965, Kurepa [5] gave a positive answer to Halperin's problem for quasiquadratic functionals defined on a vector space $V$ over $\mathbb{F} \in\{\mathbb{C}, \mathbb{H}\}$. In 1984, Vukman [9] posed the problem of representability of quasi-quadratic functionals by sesquilinear ones on modules over complex *-algebras. This problem was treated in [6-11]. The complete solution was given in [7]. It was proved that if $Q$ is a quasi-quadratic functional on a module over a complex *-algebra with an identity element, ther the mapping $S$ defined by

$$
S(x, y)=\frac{1}{4}(Q(x+y)-Q(x-y))+\frac{i}{4}(Q(x+i y)-Q(x-i y))
$$

is the unique sesquilinear functional satisfying $Q(x)=S(x, x)$. This result is an extension of the Jordan-von Neumann theorem [3] which characterises pre-Hilbert space among all normed spaces.

A mapping $J$ defined on a $*$-ring $R$ is called a Jordan $*$-derivation if it is additive and satisfies

$$
J\left(a^{2}\right)=a J(a)+J(a) a^{*} .
$$

We shall denote by $\mathcal{J}$ the set of all Jordan $*$-derivations on $R$. Over a commutative ring with the trivial involution in which 2 is not a zero divisor, the set of all Jordan $*$-derivations is equal to the set of all additive derivations [1]. A mapping $J_{a}: R \rightarrow R, a \in R$, defined by $J_{a}(b)=b a-a b^{*}$ will be called an inner Jordan $*$-derivation. In [8] it was proved that the representability of quasi-quadratic functionals by sesquilinear functionals on modules over a real Banach *-algebra $A$ with an identity element depends on the existence of Jordan *-derivations on $A$ which are not inner. The proof of this result given in [8] uses the fact that Banach algebras have enough invertible elements. It is the purpose of this note to extend this result to quasi-quadratic functionals 
defined on modules over arbitrary $*$-rings. In this general setting it is impossible to find a relation (similar to (5) in the commutative case) telling us how to recover from a quadratic functional $Q$ a sesquilinear functional $S$ satisfying $Q(x)=S(x, x)$.

\section{STATEMENT OF THE RESUlts}

Main Theorem. Let $R$ be a $*$-ring with identity 1 such that 2 is a unit in $R$. Assume that for every Jordan *-derivation $J: R \rightarrow R$ there exists a unique $a \in R$ such that $J(b)=J_{a}(b)=b a-a b^{*}, b \in R$. Then every quasi-quadratic functional $Q$ defined on an arbitrary unitary $R$-module $M$ is a quadratic functional.

Note that the uniqueness of $a$ in the above theorem is equivalent to the statement that $b a-a b^{*}=0$ for all $b \in R$ implies $a=0$. For the proof of the Main Theorem we shall need the following simple lemma.

Lemma 1. Let $R$ be $a *$-ring with identity 1 such that $b a-a b^{*}=0$ for all $b \in R$ implies $a=0$. If $e_{i}, i=1,2,3,4$, are elements from $R$ such that

$$
a e_{1} a^{*}+a e_{2} b^{*}+b e_{3} a^{*}+b e_{4} b^{*}=0
$$

for all $a, b \in R$ then $e_{i}=0, i=1,2,3,4$.

The next theorem shows that the existence of noninner Jordan $*$-derivations yields the existence of quasi-quadratic functionals that cannot be represented by sesquilinear ones.

Theorem 2. Let $R$ be a $*$-ring with identity 1 such that 2 is not a zero divisor. If $J: R \rightarrow R$ is a Jordan $*$-derivation then the mapping $Q: R \times R \rightarrow R$ given by $Q((a, b))=J(b a)-b J(a)-J(a) b^{*}$ is a quasi-quadratic functional. If $J$ is not inner then $Q$ is not a quadratic functional.

A ring $R$ is said to be a prime ring if $a R b=\{0\}$ implies $a=0$ or $b=0$. We shall prove that the mapping $F: R \rightarrow \mathcal{J}, F(a)=J_{a}$, is one-to-one if $R$ is a noncommutative prime ring. Thus, we shall prove the following result.

Corollary 3. Let $R$ be a noncommutative prime $*$-ring with identity 1 such that 2 is a unit in $R$. Then all Jordan $*$-derivations on $R$ are inner if and only if every quasi-quadratic functional $Q$ defined on an arbitrary unitary $R$-module $M$ is a quadratic functional.

Next, we shall show that all the assumptions of the Main Theorem are satisfied if $R$ is a complex $*$-algebra with an identity element. This together with the Main Theorem implies the following extension of the Jordan-von Neumann characterization of pre-Hilbert spaces (see [7]).

Corollary 4. Let $R$ be a complex *-algebra with identity 1 and let $M$ be a unitary $R$-module. Assume that $Q: M \rightarrow R$ is a quasi-quadratic functional. Under these conditions the mapping $S: M \times M \rightarrow R$ defined by the relation (6) is the unique sesquilinear functional satisfying $Q(x)=S(x, x)$.

We shall conclude by giving an example of a Jordan $*$-derivation which is not inner.

Example 5. There exists a Jordan $*$-derivation on a finite-dimensional noncommutative real $*$-algebra with an identity element which is not inner. 


\section{Proofs}

Proof of Main Theorem. Let $Q$ be a quasi-quadratic functional defined on a unitary $R$-module $M$. We shall divide our proof into two steps. First, we shall prove that if the restriction of $Q$ to each submodule of $M$ generated by two elements is a quadratic functional, then $Q$ is a quadratic functional on $M$. Our second step will be to prove that under the assumptions of the Main Theorem every quasi-quadratic functional defined on an arbitrary unitary $R$-module $M$ generated by two elements is a quadratic functional.

Step 1. Assume that the restriction of $Q$ to each submodule of $M$ generated by two elements is a quadratic functional. Let us choose arbitrary elements $x, y \in M$. We denote by $M_{x, y}=\{a x+b y: a, b \in R\}$ the submodule of $M$ generated by $x$ and $y$. According to our assumption there exists a sesquilinear functional $S_{x, y}: M_{x, y} \times M_{x, y} \rightarrow R$ such that

$$
\begin{aligned}
Q(a x+b y)= & S_{x, y}(a x+b y, a x+b y) \\
= & a S_{x, y}(x, x) a^{*}+a S_{x, y}(x, y) b^{*} \\
& +b S_{x, y}(y, x) a^{*}+b S_{x, y}(y, y) b^{*}, \quad a, b \in R .
\end{aligned}
$$

Let us define a functional $S: M \times M \rightarrow R$ by $S(x, y)=S_{x, y}(x, y)$ for all $x, y \in M$.

In order to see that the mapping $S$ is well defined we assume that there exists another sesquilinear functional $T_{x, y}: M_{x, y} \times M_{x, y} \rightarrow R$ satisfying

$$
\begin{aligned}
Q(a x+b y)= & T_{x, y}(a x+b y, a x+b y) \\
= & a T_{x, y}(x, x) a^{*}+a T_{x, y}(x, y) b^{*} \\
& +b T_{x, y}(y, x) a^{*}+b T_{x, y}(y, y) b^{*}, \quad a, b \in R .
\end{aligned}
$$

Comparing this with (8) and using Lemma 1 we get that $S_{x, y}(x, y)=$ $T_{x, y}(x, y)$. Thus, $S$ is well defined. Moreover, we have proved that

$$
S_{y, x}(x, y)=S_{x, y}(x, y)
$$

holds for all $x, y \in M$. Let $x, y$, and $z$ be elements from $M$. Then we have $S_{x, y}(x, x)=Q(1 x+0 y)=Q(1 x+0 z)=S_{x, z}(x, x)$. In particular, we obtain $S_{x, x}(x, x)=S_{x, y}(x, x)$. This last relation implies together with (9) that (8) can be rewritten as

$$
\begin{aligned}
& Q(a x+b y)=a S(x, x) a^{*}+a S(x, y) b^{*} \\
& +b S(y, x) a^{*}+b S(y, y) b^{*}, \quad a, b \in R,
\end{aligned}
$$

where $x, y$ are arbitrary elements from $M$. It follows that $Q(x)=S(x, x)$ is valid for all $x \in M$. In order to complete the first step of our proof we must show that $S$ is a sesquilinear functional.

For arbitrary $x, y \in M$ and $a, b, c, d \in R$ we have

$$
\begin{gathered}
c a S(x, x) a^{*} c^{*}+c a S(x, y) b^{*} d^{*}+d b S(y, x) a^{*} c^{*}+d b S(y, y) b^{*} d^{*} \\
=Q(c a x+d b y)= \\
+S(a x, a x) c^{*}+c S(a x, b y) d^{*} \\
+d S(b y, a x) c^{*}+d S(b y, b y) d^{*} .
\end{gathered}
$$

Applying Lemma 1 we get $S(a x, b y)=a S(x, y) b^{*}$. It remains to prove that $S$ is biadditive. Define

$$
b_{1}=Q\left(a_{1} x_{1}+a_{2} x_{2}+a_{3} x_{3}\right), \quad b_{2}=Q\left(a_{1} x_{1}+a_{2} x_{2}-a_{3} x_{3}\right),
$$


and

$$
b_{3}=Q\left(a_{1} x_{1}-a_{2} x_{2}-a_{3} x_{3}\right) .
$$

The parallelogram law (3) gives us

$$
\begin{aligned}
b_{1}+b_{2} & =2 Q\left(a_{1} x_{1}+a_{2} x_{2}\right)+2 Q\left(a_{3} x_{3}\right), \\
-b_{2}-b_{3} & =-2 Q\left(a_{1} x_{1}-a_{3} x_{3}\right)-2 Q\left(a_{2} x_{2}\right), \\
b_{1}+b_{3} & =2 Q\left(a_{1} x_{1}\right)+2 Q\left(a_{2} x_{2}+a_{3} x_{3}\right) .
\end{aligned}
$$

Solving this system of equations and using (10) we obtain

$$
b_{1}=\sum_{i, j=1}^{3} a_{i} S\left(x_{i}, x_{j}\right) a_{j}^{*} \text {. }
$$

In particular, for arbitrary $x, y, z \in M$ and $a, b \in R$ we have the relation

$$
\begin{aligned}
Q(a x+a y+b z)= & a(S(x, x)+S(y, x)+S(x, y)+S(y, y)) a^{*} \\
& +a(S(x, z)+S(y, z)) b^{*} \\
& +b(S(z, x)+S(z, y)) a^{*}+b S(z, z) b^{*} .
\end{aligned}
$$

On the other hand, using (10) we get that

$$
\begin{aligned}
Q(a(x+y)+b z)= & a S(x+y, x+y) a^{*}+a S(x+y, z) b^{*} \\
& +b S(z, x+y) a^{*}+b S(z, z) b^{*} .
\end{aligned}
$$

Comparing the two expressions for $Q(a x+a y+b z)$ we obtain, using Lemma 1 , the biadditivity of $S$. Thus, under the assumptions of the Main Theorem, a quasi-quadratic functional $Q$ on $M$ is a quadratic functional if and only if its restriction to each submodule generated by two elements is a quadratic functional.

Step 2. Let $M=\{a x+b y: a, b \in R\}$ be a unitary $R$-module generated by $x$ and $y$. We have to prove that for a given quasi-quadratic functional $Q: M \rightarrow R$ there exists a sesquilinear functional $S$ from $M \times M$ to $R$ such that $Q(z)=S(z, z)$ for all $z \in M$.

Let us define a functional $D: R \times R \rightarrow R$ by

$$
D(a, b)=Q(a x+b y)-a Q(x) a^{*}-b Q(y) b^{*}-2^{-1}\left(a f b^{*}+b f a^{*}\right),
$$

where $f=Q(x+y)-Q(x)-Q(y)$. We shall first prove that $D$ is biadditive. Clearly, it is enough to prove that the functional $E$ given by $E(a, b)=$ $Q(a x+b y)-a Q(x) a^{*}-b Q(y) b^{*}$ is biadditive. Applying the parallelogram law (3) we get

$$
\begin{aligned}
2 E(a & , b)+2 E(c, b) \\
& =2 Q(a x+b y)+2 Q(c x+b y)-2 Q(a x)-2 Q(c x)-4 Q(b y) \\
& =Q((a+c) x+2 b y)+Q((a-c) x)-2 Q(a x)-2 Q(c x)-Q(2 b y) \\
& =Q((a+c) x+2 b y)-Q((a+c) x)-Q(2 b y)=E(a+c, 2 b) .
\end{aligned}
$$

Substituting $c=0$ and using the obvious relation $E(0, b)=0$ we obtain

$$
2 E(a, b)=E(a, 2 b) \text {. }
$$

It follows from (12) and (13) that the mapping $E$ is additive in the first argument. The same must be true for the functional $D$. In the same way we prove that $D$ is additive in the second argument. 
It is not difficult to verify that (4) and (11) imply

$$
D(a, a)=0, \quad a \in R,
$$

and

$$
D(c a, c b)=c D(a, b) c^{*}, \quad a, b, c \in R .
$$

Using these two relations and biadditivity of $D$ we shall prove that the mapping $J: R \rightarrow R$ given by $J(a)=D(a, 1)$ is a Jordan $*$-derivation satisfying

$$
D(a, b)=J(a b)-a J(b)-J(b) a^{*}, \quad a, b \in R .
$$

Clearly, $J$ is additive. For arbitrary $a, b, c, d \in R$ we have

$$
\begin{aligned}
& a D(b, c) a^{*}+D(d b, a c)+D(a b, d c)+d D(b, c) d^{*} \\
& \quad=D((a+d) b,(a+d) c)=(a+d) D(b, c)(a+d)^{*} \\
& \quad=a D(b, c) a^{*}+d D(b, c) a^{*}+a D(b, c) d^{*}+d D(b, c) d^{*},
\end{aligned}
$$

which yields

$$
D(d b, a c)+D(a b, d c)=d D(b, c) a^{*}+a D(b, c) d^{*} .
$$

Putting $c=d=1$ we get $D(b, a)+J(a b)=J(b) a^{*}+a J(b)$. As $D(a, a)=0$ implies $D(a, b)=-D(b, a)$, we have proved that (14) is valid. Replacing $a$ in this relation by $b a$ we see that

$$
b J(a) b^{*}=J(b a b)-b a J(b)-J(b) a^{*} b^{*}
$$

holds for all $a, b \in R$. Putting $a=1$ and using $J(1)=0$ we finally get $J\left(b^{2}\right)=b J(b)+J(b) b^{*}$ for all $b \in R$.

According to our assumptions, $J$ is an inner Jordan *-derivation. Thus, we can find an element $g \in R$ such that $J(a)=a g-g a^{*}$ is valid for all $a \in R$. It follows from (14) that

$$
D(a, b)=a g b^{*}-b g a^{*}, \quad a, b \in R .
$$

Applying (11) one can easily see that

$$
Q(a x+b y)=a e_{11} a^{*}+a e_{12} b^{*}+b e_{21} a^{*}+b e_{22} b^{*}, \quad a, b \in R,
$$

where $e_{11}=Q(x), e_{12}=g+2^{-1} f, e_{21}=2^{-1} f-g$, and $e_{22}=Q(y)$. We define $S: M \times M \rightarrow R$ by

$S(a x+b y, c x+d y)=a e_{11} c^{*}+a e_{12} d^{*}+b e_{21} c^{*}+b e_{22} d^{*}, \quad a, b, c, d \in R$.

In order to see that $S$ is well defined we choose $a_{1}, a_{2} \in R$ such that $a_{1} x+$ $a_{2} y=0$. For arbitrary elements $b_{1}, b_{2} \in R$ we have

$$
\begin{aligned}
\sum_{i, j=1}^{2} b_{i} e_{i j} b_{j}^{*} & =Q\left(b_{1} x+b_{2} y\right)=Q\left(\left(a_{1}+b_{1}\right) x+\left(a_{2}+b_{2}\right) y\right) \\
& =\sum_{i, j=1}^{2}\left(a_{i}+b_{i}\right) e_{i j}\left(a_{j}^{*}+b_{j}^{*}\right) \\
& =\sum_{i, j=1}^{2} a_{i} e_{i j} a_{j}^{*}+\sum_{i, j=1}^{2} a_{i} e_{i j} b_{j}^{*}+\sum_{i, j=1}^{2} b_{i} e_{i j} a_{j}^{*}+\sum_{i, j=1}^{2} b_{i} e_{i j} b_{j}^{*} .
\end{aligned}
$$


It follows from $0=Q\left(a_{1} x+a_{2} y\right)=\sum_{i, j=1}^{2} a_{i} e_{i j} a_{j}^{*}$ that

$$
\sum_{i, j=1}^{2} a_{i} e_{i j} b_{j}^{*}+\sum_{i, j=1}^{2} b_{i} e_{i j} a_{j}^{*}=0 .
$$

Putting $b_{1}=1$ and $b_{2}=0$ we get $p+q=0$, where

$$
p=a_{1} e_{11}+a_{2} e_{21}, \quad q=e_{11} a_{1}^{*}+e_{12} a_{2}^{*} .
$$

On the other hand, if we set in (15) $b_{1}=c$ and $b_{2}=0$, we obtain $p c^{*}+c q=0$. Together with $c q+c p=0$ this implies $c p-p c^{*}=0$ for all $c \in A$. It follows that $p=q=0$, or

$$
S\left(a_{1} x+a_{2} y, x\right)=0=S\left(x, a_{1} x+a_{2} y\right) .
$$

In a similar way we get

$$
S\left(a_{1} x+a_{2} y, y\right)=0=S\left(y, a_{1} x+a_{2} y\right) .
$$

Thus, $S$ is well defined. Clearly, it is a sesquilinear functional satisfying $Q(z)=$ $S(z, z)$ for all $z \in M$. This completes the proof.

Proof of Lemma 1. Putting $a=1$ and $b=0$ we get $e_{1}=0$. Similarly, we obtain $e_{4}=0$. Substituting $a=b=1$ we see that $e_{2}=-e_{3}$. Substituting once again $b=1$ we get that $a e_{2}-e_{2} a^{*}=0$ is valid for all $a \in R$. Thus, $e_{2}=e_{3}=0$. This completes the proof.

Proof of Theorem 2. It is easy to verify that $Q$ satisfies the parallelogram law (3). In order to see that also the homogeneity law (4) is fulfilled we must show that every Jordan $*$-derivation $J: R \rightarrow R$ satisfies

$$
J(c b c a)=c b J(c a)+J(c a) b^{*} c^{*}+c J(b a) c^{*}-c b J(a) c^{*}-c J(a) b^{*} c^{*}
$$

for all $a, b, c \in R$. For this purpose first replace $a$ by $a+b$ in (7) to get

$$
J(a b)+J(b a)=b J(a)+a J(b)+J(a) b^{*}+J(b) a^{*}
$$

for all $a, b \in R$. Consider now $d=J(a(a b+b a)+(a b+b a) a)$. Using (17) we see that

$$
\begin{aligned}
d= & a J(a b+b a)+(a b+b a) J(a)+J(a b+b a) a^{*}+J(a)\left(b^{*} a^{*}+a^{*} b^{*}\right) \\
= & 2 a b J(a)+a^{2} J(b)+a J(a) b^{*}+2 a J(b) a^{*}+b a J(a) \\
& +b J(a) a^{*}+2 J(a) b^{*} a^{*}+J(b) a^{* 2}+J(a) a^{*} b^{*} .
\end{aligned}
$$

On the other hand,

$$
\begin{aligned}
d & =2 J(a b a)+J\left(a^{2} b\right)+J\left(b a^{2}\right) \\
& =2 J(a b a)+b J\left(a^{2}\right)+a^{2} J(b)+J\left(a^{2}\right) b^{*}+J(b) a^{* 2} \\
& =2 J(a b a)+b a J(a)+b J(a) a^{*}+a^{2} J(b)+a J(a) b^{*}+J(a) a^{*} b^{*}+J(b) a^{* 2} .
\end{aligned}
$$

Comparing the two expressions for $d$ we arrive at

$$
J(a b a)=J(a) b^{*} a^{*}+a J(b) a^{*}+a b J(a), \quad a, b \in R .
$$

Replacing $a$ in (18) by $a+c$ we obtain

$$
\begin{aligned}
J(a b c+c b a)= & J(a) b^{*} c^{*}+a J(b) c^{*}+a b J(c)+J(c) b^{*} a^{*} \\
& +c J(b) a^{*}+c b J(a), \quad a, b, c \in R .
\end{aligned}
$$


Applying (18) and (19) we get

$$
\begin{aligned}
J(c b c a) & =J(c b(c a)+(c a) b c)-J(c(a b) c) \\
& =c b J(c a)+J(c a) b^{*} c^{*}+c\left(J(b) a^{*}+a J(b)-J(a b)\right) c^{*} .
\end{aligned}
$$

Applying (17) we get (16). Thus, we have proved that $Q$ is a quasi-quadratic functional.

Assume now that $J$ is not inner. If there is a sesquilinear functional $S$ which generates $Q$, then $S$ is of the form $S((a, b),(c, d))=a e d^{*}+b f c^{*}$ for some $e, f \in R$. The relation $Q((a, b))=S((a, b),(a, b))$ with $b=1$ gives us $J(a)=-a e-f a^{*}$. Since $J(1)=0$, we have $e=-f$, so that $J$ is an inner Jordan $*$-derivation. This contradiction completes the proof.

Proof of Corollary 3. Let us first assume that all Jordan $*$-derivations on $R$ are inner. We claim that $J_{a}=0, a \in R$, implies $a=0$. Indeed, for such an $a$ we have

$$
b a=a b^{*}
$$

for all $b \in R$. Replacing $b$ by $b c$ and applying (20) two times we get

$$
(b c-c b) a=0 .
$$

Substituting $c=d c$ in (21) we obtain $(b d c-d c b) a=0$, which can be rewritten as

$$
(b d-d b) c a+d(b c-c b) a=0
$$

where $b, c, d$ are arbitrary elements from $R$. The second term is zero by (21). As $R$ is noncommutative and prime, we have necessarily $a=0$. Using the Main Theorem one can complete the proof of the "if part". Theorem 2 shows that the converse is also true.

Proof of Corollary 4. Substituting $a=i a$ and $b=i$ in (17) we prove that every Jordan *-derivation on $R$ is inner. From $J_{a}(i)=2 i a$ it follows that $a \neq 0$ implies that $J_{a}$ is nonzero. Using the Main Theorem one can complete the proof.

Verification of Example 5. Let $R$ be a real *-algebra consisting of elements $\lambda+u \mu$, where $\lambda$ and $\mu$ are complex numbers. We define the operations by $t(\lambda+u \mu)=t \lambda+u(t \mu)$ for real $t,\left(\lambda_{1}+u \mu_{1}\right)+\left(\lambda_{2}+u \mu_{2}\right)=\left(\lambda_{1}+\lambda_{2}\right)+u\left(\mu_{1}+\mu_{2}\right)$, $\left(\lambda_{1}+u \mu_{1}\right)\left(\lambda_{2}+u \mu_{2}\right)=\lambda_{1} \lambda_{2}+u\left(\mu_{1} \lambda_{2}+\overline{\lambda_{1}} \mu_{2}\right)$ and the involution by $(\lambda+u \mu)^{*}=$ $\bar{\lambda}-u \mu$.

There exists a nontrivial and therefore discontinuous additive derivation on $\mathbb{R}$, that is, an additive function $f: \mathbb{R} \rightarrow \mathbb{R}$ satisfying $f(t s)=t f(s)+s f(t)$ for all pairs $t, s \in \mathbb{R}$ (see [12]). Putting $D(s+i t)=f(s)-i f(t)$ we get a function $D: \mathbb{C} \rightarrow \mathbb{C}$ which is additive and satisfies $D\left(\lambda^{2}\right)=2 \bar{\lambda} D(\lambda)$. It is not difficult to verify that the mapping $J: R \rightarrow R$ given by $J(\lambda+u \mu)=u D(\lambda)$ is a Jordan $*$-derivation. However, it is discontinuous and therefore noninner.

\section{ACKNOWLEDGMENT}

I am indebted to my colleague M. Brešar for his valuable suggestions. 


\section{REFERENCES}

1. T. M. K. Davison, Jordan derivations and quasi-bilinear forms, Comm. Algebra 12 (1984), 23-32.

2. A. M. Gleason, The definition of a quadratic form, Amer. Math. Monthly 73 (1966), 1049-1056.

3. P. Jordan and J. von Neumann, On inner products in linear metric space, Ann. of Math. (2) 36 (1935), 719-723.

4. S. Kurepa, The Cauchy functional equation and scalar product in vector spaces, Glas. Mat. Ser. III 19 (1964), 23-35.

5. __, Quadratic and sesquilinear functionals, Glas. Mat. Ser. III 20 (1965), 79-92.

6. P. Šemrl, On quadratic and sesquilinear functionals, Aequationes Math. 19 (1986), 184-190.

7. _ـ On quadratic functionals, Bull. Austral. Math. Soc. 37 (1988), 27-29.

8. __ Quadratic functionals and Jordan *-derivations, Studia Math. 97 (1991), 157-165.

9. J. Vukman, A result concerning additive functions in hermitian Banach *-algebras and an application, Proc. Amer. Math. Soc. 91 (1984), 367-372.

10. Some results concerning the Cauchy functional equation in certain Banach algebras, Bull. Austral. Math. Soc. 31 (1985), 137-144.

11. __ Some functional equations in Banach algebras and an application, Proc. Amer. Math. Soc. 100 (1987), 133-136.

12. O. Zariski and P. Sammuel, Commutative algebra, Van Nostrand, Princeton, NJ, 1958.

Department Of Mathematics, University of Ljubljana, JadRanska 19, 61000 Ljubljana, SLOVENIA

E-mail address: peter.semrlouni-lj.si 